\title{
Visualizing Poetry with SPARSAR - Visual Maps from Poetic Content
}

\author{
Rodolfo Delmonte \\ Department of Language Studies \& Department of Computer Science \\ Ca' Foscari University - 30123, Venezia, Italy \\ delmont@unive.it
}

\begin{abstract}
In this paper we present a specific application of SPARSAR, a system for poetry analysis and TextToSpeech "expressive reading". We will focus on the graphical output organized at three macro levels, a Phonetic Relational View where phonetic and phonological features are highlighted; a Poetic Relational View that accounts for a poem rhyming and metrical structure; and a Semantic Relational View that shows semantic and pragmatic relations in the poem. We will also discuss how colours may be used appropriately to account for the overall underlying attitude expressed in the poem, whether directed to sadness or to happiness. This is done following traditional approaches which assume that the underlying feeling of a poem is strictly related to the sounds conveyed by the words besides their meaning. This will be shown using part of Shakespeare's Sonnets.
\end{abstract}

\section{$1 \quad$ Introduction}

The contents of a poem cover many different fields from a sensorial point of view to a mental and a auditory linguistic one. A poem may please our hearing for its rhythm and rhyme structure, or simply for the network of alliterations it evokes be they consonances or assonances; it may attract our attention for its structure of meaning, organized on a coherent lattice of anaphoric and coreferential links or suggested and extracted from inferential and metaphorical links to symbolic meanings obtained by a variety of rhetorical devices. Most if not all of these facets of a poem are derived from the analysis of SPARSAR, the system for poetry analysis which has been presented to a number of international conferences (Delmonte 2013a; 2013b; 2014) - and to Demo sessions in its TextToSpeech "expressive reading" version (Delmonte \& Bacalu, 2012; Delmonte \& Prati, 2014; Delmonte, 2015).
Most of a poem's content can be captured considering three basic views on the poem itself: one that covers what can be called the overall sound pattern of the poem - and this is related to the phonetics and the phonology of the words contained in the poem - Phonetic Relational View. Another view is the one that captures the main poetic devices related to rhythm, that is the rhyme structure and the metrical structure - this view will be called Poetic Relational View. Finally, the semantic and pragmatic contents of the poem which are related to relations entertained by predicates and arguments expressed in the poem, relations at lexical semantic level, relations at metaphorical and anaphoric level - this view will be called Semantic Relational View.

In this paper we will concentrate on the three views above, which are visualized by the graphical output of the system and has been implemented by extracting the various properties and features of the poem and are analyzed in ten separate poetic maps. These maps are organized as follows:

* A General Description map including seven Macro Indices with a statistical evaluation of such descriptors as: Semantic Density Evaluation; General Poetic Devices; General Rhetoric Devices etc., Prosodic Distribution; Rhyming Schemes; Metrical Structure. This map is discussed and presented in previous publications, so I will not show it here;

* Phonetic Relational Views: five maps,

- Assonances, i.e. all vowels contained in stressed vowel nuclei which have been repeated in the poem within a certain interval - not just in adjacency;

- Consonances, i.e. all consonant onsets of stressed syllables again repeated in the poem within a certain interval;

- All word repetitions, be it stressed or unstressed; 
○ one for the Unvoiced/Voiced opposition as documented in syllable onset of stressed words (stress demotion counts as unstressed);

- another for a subdivision of all consonant syllable onsets, including consonant cluster onsets, and organized in three main phonological classes:

- Continuants (only fricatives);

- Obstruents (Plosives and Affricates);

- Sonorants (Liquids, Vibrants, Approximants; Glides; Nasals).

* Poetic Relation Views:

- Metrical Structure, Rhyming Structure and Expected Acoustic Length all in one single map.

Semantic Relational View: four maps,

- A map including polarity marked words (Positive vs Negative) and words belonging to Abstract vs Concrete semantic class ${ }^{1}$;

- A map including polarity marked words (Positive vs Negative) and words belonging to Eventive vs State semantic class;

- A map including Main Topic words; Anaphorically linked words; Inferentially linked words; Metaphorically linked words i.e. words linked explicitly by "like" or "as", words linked by recurring symbolic meanings (woman/serpent or woman/moon or woman/rose);

- A map showing predicate argument relations intervening between words, marked at core argument words only, indicating predicate and semantic role; eventive anaphora between verbs.

Graphical maps highlight differences using colours. The use of colours associated to sound in poetry has a long tradition. Rimbaud composed a poem devoted to "Vowels" where colours where specifically associated to each of the main five vowels. Roman Jakobson wrote extensively about sound and colour in a number of papers (1976;

\footnotetext{
${ }^{1}$ see in particular Brysbaert et al. 2014 that has a database of $40 \mathrm{~K}$ entries. We are also using a manually annotated lexicon of $10 \mathrm{~K}$ entries and WordNet supersenses or broad semantic classes. We are not using MRCDatabase which only has some 8,000 concrete + some 9,000 imagery classified entries because it is difficult to adapt and integrated into our system.
}

Jakobson \& Waugh, 1978:188; lately Mazzeo, 2004). As Tsur (1992) notes, Fónagy in 1961 wrote an article in which he connected explicitly the use of certain types of consonant sounds associated to certain moods: unvoiced and obstruent consonants are associated with aggressive mood; sonorants with tender moods. Fónagy mentioned the work of M.Macdermott (1940) who in her study identified a specific quality associated to "dark" vowels, i.e. back vowels, that of being linked with dark colours, mystic obscurity, hatred and struggle. For this reason, we then decided to evaluate all information made available by SPARSAR at the three macro levels in order to check these findings about the association of mood and sound. This will be discussed in a final section of the paper devoted to correlations in Shakespeare's sonnets.

As a result, we will also be using darker colours for highlighting back and front vowels as opposed to low and middle vowels, these latter with light colours. The same will apply to representing unvoiced and obstruent consonants as opposed to voiced and sonorants. But as Tsur (1992:15) notes, this sound-colour association with mood or attitude has no real significance without a link to semantics. In the Semantic Relational View, we will be using dark colours for Concrete referents vs Abstract ones with lighter colours; dark colours also for Negatively marked words as opposed to Positively marked ones with lighter colours. The same strategy will apply to other poetic maps: this technique has certainly the good quality of highlighting opposing differences at some level of abstraction $^{2}$.

The usefulness of this visualization is intuitively related to various potential users and for different purposes. First of all, translators of poetry would certainly benefit from the decomposition of the poem and the fine-grained analysis, in view of the need to preserve as much as possible of the original qualities of the source poem in the target language. Other possible users are literary critics and literature teachers at various levels. Graphical output is essentially produced to allow immediate and direct comparison between different poems

2 our approach is not comparable to work by Saif Mohammad (2011a;2011b), where colours are associated with words on the basis of what their mental image may suggest to the mind of annotators hired via Mechanical Turk. The resource only contains word-colour association for some 12,000 entries over the $27 \mathrm{~K}$ items listed. 
and different poets. In order to show the usefulness and power of these visualization, I have chosen two different English poets in different time periods: Shakespeare with Sonnet 1 and 60; and Sylvia Plath, with Edge.

The paper is organized as follows: a short state of the art in the following section; then the views of three poems accompanied by comments; some conclusion.

\section{Related Work}

Computational work on poetry addresses a number of subfields which are however strongly related. They include automated annotation, analysis, or translation of poetry, as well as poetry generation, that we comment here below. Other common subfields regard automatic grapheme-to-phoneme translation for out of vocabulary words as discussed in (Reddy \& Goldsmith, 2010). Genzel et al. (2010) use CMU pronunciation dictionary to derive stress and rhyming information, and incorporate constraints on meter and rhyme into a machine translation system. There has also been some work on computational approaches to characterizing rhymes (Byrd and Chodorow, 1985) and global properties of the rhyme network (Sonderegger, 2011) in English.

Green et al. (2010) use a finite state transducer to infer the syllable-stress assignments in lines of poetry under metrical constraints. They contribute variations similar to the schemes below, by allowing an optional inversion of stress in the iambic foot. This variation is however only motivated by heuristics, noting that "poets often use the word 'mother' ( $\mathrm{S}^{*} \mathrm{~S}$ ) at the beginnings and ends of lines, where it theoretically should not appear." So eventually, there is no control of the internal syntactic or semantic structure of the newly obtained sequence of feet: the optional change is only positionally motivated. They employ statistical methods to analyze, generate, and translate rhythmic poetry. They first apply unsupervised learning to reveal word-stress patterns in a corpus of raw poetry. They then use these word-stress patterns, in addition to rhyme and discourse models, to generate English love poetry. Finally, they translate Italian poetry into English, choosing target realizations that conform to desired rhythmic patterns. They, however, concentrate on only one type of poetic meter, the iambic pentameter. And they use the audio transcripts - made by just one person - to create the syllable-based word-stress gold standard corpus for testing, made of some 70 lines taken from Shakespeare's sonnets. Audio transcripts without supporting acoustic analysis ${ }^{3}$ are not always the best manner to deal with stress assignment in syllable positions which might or might not conform to a strict sequence of iambs. There is no indication of what kind of criteria have been used, and it must be noted that the three acoustic cues may well not be congruent (see Tsur, 2014). So eventually results obtained are rather difficult to evaluate. As the authors note, spoken recordings may contain lexical stress reversals and archaic pronunciations ${ }^{4}$. Their conclusion is that "this useful information is not available in typical pronunciation dictionaries". Further on, (p. 531) they comment "the probability of stressing 'at' is $40 \%$ in general, but this increases to $91 \%$ when the next word is 'the'. " We assume that demoting or promoting word stress requires information which is context and syntactically dependent. Proper use of one-syllable words remains tricky. In our opinion, machine learning would need much bigger training data than the ones used by the authors for their experiment.

There's a large number of papers on poetry generation starting from work documented in a number of publications by P. Gérvas $(2001 ; 2010)$ who makes use of Case Based Reasoning to induce the best line structure. Other interesting attempts are by Toivonen et al.(2012) who use a corpusbased approach to generate poetry in Finnish. Their idea is to contribute the knowledge needed in content and form by two separate corpora, one providing semantic content, and another for grammatical and poetic structure. Morphological analysis and synthesis is used together with textmining methods. Basque poetry generation is the

\footnotetext{
${ }^{3}$ One questions could be "Has the person transcribing stress pattern been using pitch as main acoustic correlate for stress position, or loudness (intensity or energy) or else durational patterns?". The choice of one or the other acoustic correlated might change significantly the final outcome.

${ }^{4}$ At p.528 they present a table where they list a number of words - partly function and partly content words - associated to probability values indicating their higher or lower propensity to receive word stress. They comment that "Function words and possessives tend to be unstressed, while content words tend to be stressed, though many words are used both ways".
} 
topic of Agirrezabal et al. 2013 paper which uses POS-tags to induce the linear ordering and WordNet to select best semantic choice in context.

Manurung et al., 2000 have explored the problem of poetry generation under some constraints using machine learning techniques. With their work the authors intended to fill the gap in the generation paradigm, and "to shed some light on what often seems to be the most enigmatic and mysterious forms of artistic expression". The conclusion they reach is that "...despite our implementation being at a very early stage, the sample output succeeds in showing how the stochastic hillclimbing search model manages to produce text that satisfies these constraints." However, when we come to the evaluation of metre we discover that they base their approach on disputable premises. The authors quote the first line of what could be a normal limerick but totally misinterpret the metrical structure. In limericks, what we are dealing with are not dactyls - TAtata but anapests, tataTA, that is a sequence of two unstressed plus a closing stressed syllable. This is a well known characteristic feature of limericks and the typical rhythm is usually preceded and introduced by a iamb "there ONCE", and followed by two anapests, "was a MAN", "from maDRAS". Here in particular it is the syntactic-semantic phrase that determines the choice of foot, and not the scansion provided by the authors ${ }^{5}$.

Reddy \& Knight (2011) produce an unsupervised machine learning algorithm for finding rhyme schemes which is intended to be language-independent. It works on the intuition that "a collection of rhyming poetry inevitably contains repetition of rhyming pairs. ... This is partly due to sparsity of rhymes - many words that have no rhymes at all, and many others have only a handful, forcing poets to reuse rhyming pairs." The authors harness this repetition to build an unsupervised algorithm to infer rhyme schemes, based on a model of stanza generation. "We test the algorithm on rhyming poetry in English and French." The definition of rhyme the authors used

\footnotetext{
5 "For instance, the line 'There /once was a /man from $\mathrm{Ma} / \mathrm{dras}^{\prime}$, has a stress pattern of (w,s,w,w,s,w,w,s). This can be divided into feet as $(\mathrm{w}),(\mathrm{s}, \mathrm{w}, \mathrm{w}),(\mathrm{s}, \mathrm{w}, \mathrm{w}),(\mathrm{s})$. In other words, this line consists of a single upbeat (the weak syllable before the first strong syllable), followed by 2 dactyls (a classical poetry unit consisting of a strong syllable followed by two weak ones), and ended with a strong beat."(ibid.7)
}

is the strict one of perfect rhyme: two words rhyme if their final stressed vowels and all following phonemes are identical. So no half rhymes are considered. Rhyming lines are checked from CELEX phonological database.

\section{Three Views via Poetic Graphical Maps}

The basic idea underlying poetic graphical maps is that of making available to the user an insight of the poem which is hardly realized even if the analysis is carried out manually by an expert literary critic. This is also due to the fact that the expertise required for the production of all the maps ranges from acoustic phonetics to semantics and pragmatics, a knowledge that is not usually possessed by a single person. All the graphical representations associated to the poems are produced by SWI Prolog, inside the system which is freely downloadable from its website, at sparsar.wordpress.com. For lack of space, we will show maps related to two of Shakespeare's Sonnets, Sonnet 1 and Sonnet 60 and compare them to Sylvia Plath's Edge, to highlight similarities and to show that the system can handle totally different poems still allowing comparisons to be made neatly. All Phonetic Views are shown in Arpabet, i.e. the computer based phonetic alphabet .

Poem and Poetic Devices :- Assonance Alliterations sonnet_1

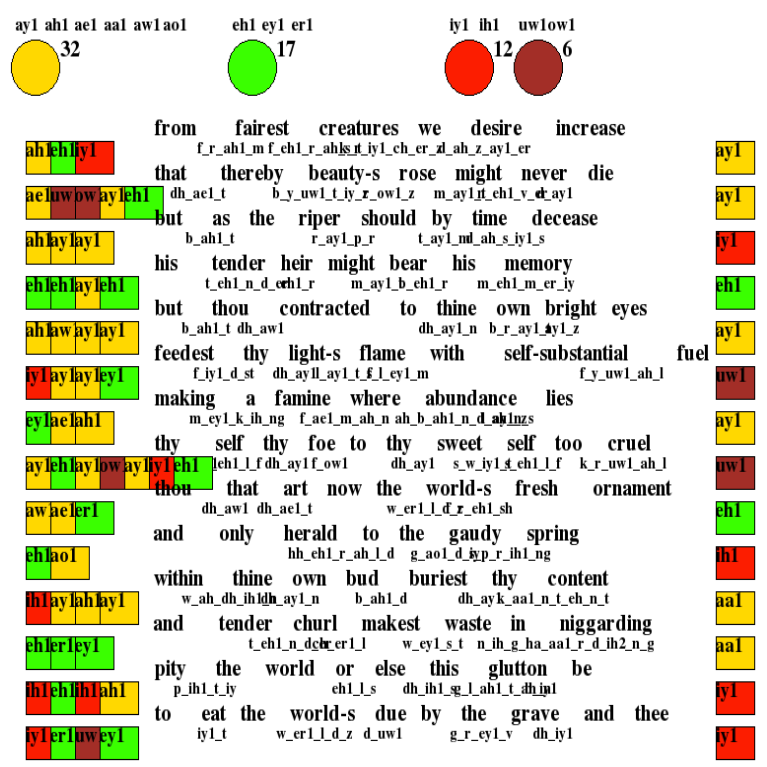




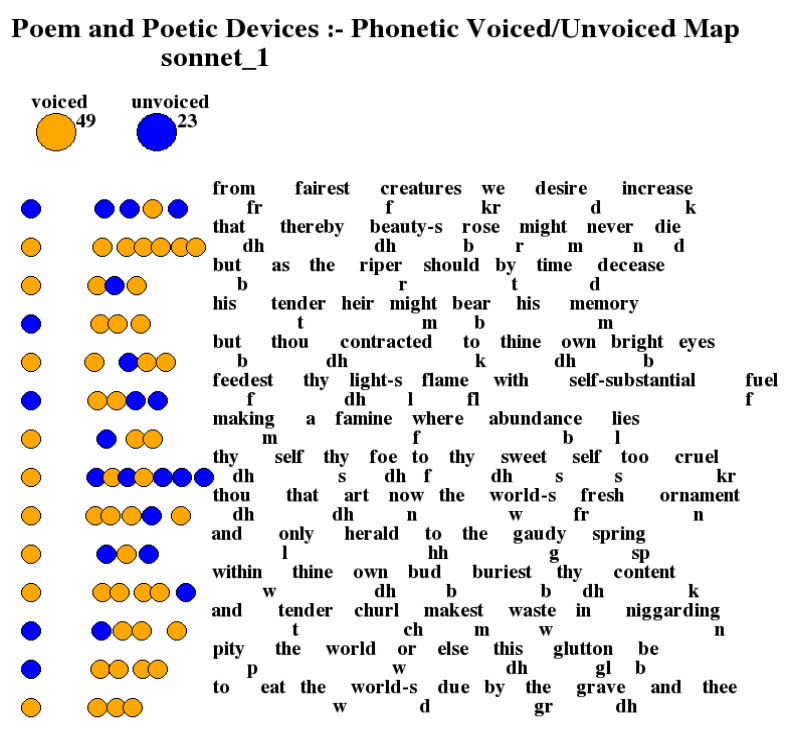

Poem and Poetic Devices :- Consonance Alliterations sonnet1

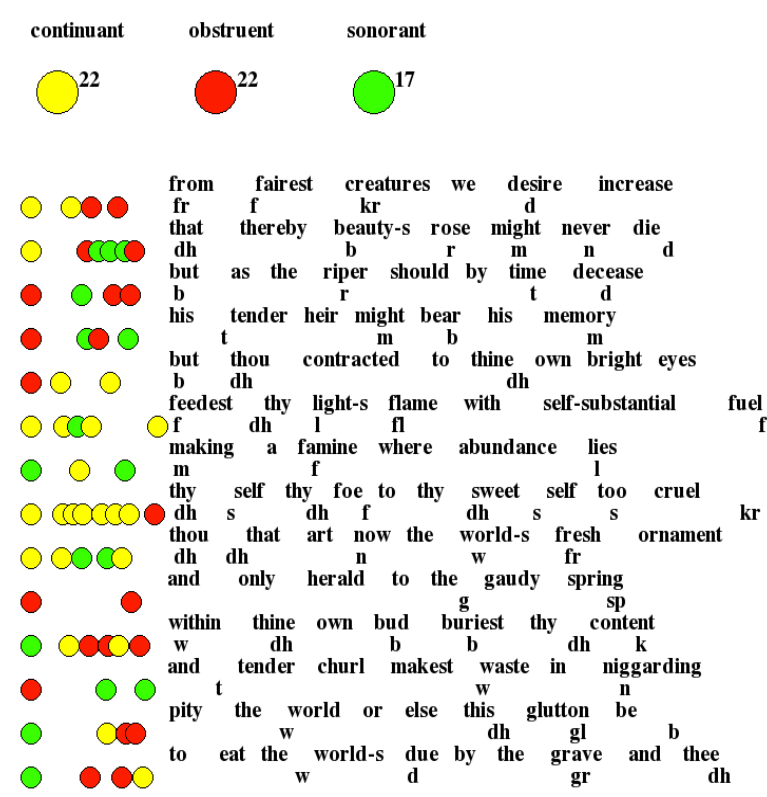

We will start commenting the Phonetic Relational View and its related maps. The first map is concerned with Assonances. Here sounds are grouped into Vowel Areas, as said above, which include also diphthongs: now, in area choice what we have considered is the onset vowel. We have disregarded the offset glide which is less persistent and might also not reach its target articulation. We will have also combined together front high vowels, which can express suffering and pain, with back dark vowels: both can be treated as marked vowels, compared to middle and low vowels ${ }^{6}$.

Assonances and Consonances are derived from syllable structure in stressed position of repeated sounds within a certain line span: in particular, Consonances are derived from syllable onset while Assonances from syllable nuclei in stressed position. The Voiced/Unvoiced View is taken from all consonant onsets of stressed words. As can be noticed from the maps above, the choice of warm colours is selected for respectively, CONTINUANT (yellow), VOICED (orange), SONORANT (green), Centre/Low Vowel Area (gold), Middle Vowel Area (green); and cold colours respectively for UNVOICED (blue), Back Vowel Area (brown). We used then red for OBSTRUENT (red), Front High Vowel Area (red), to indicate suffering and surprise associated to speech signal interruption in obstruents.

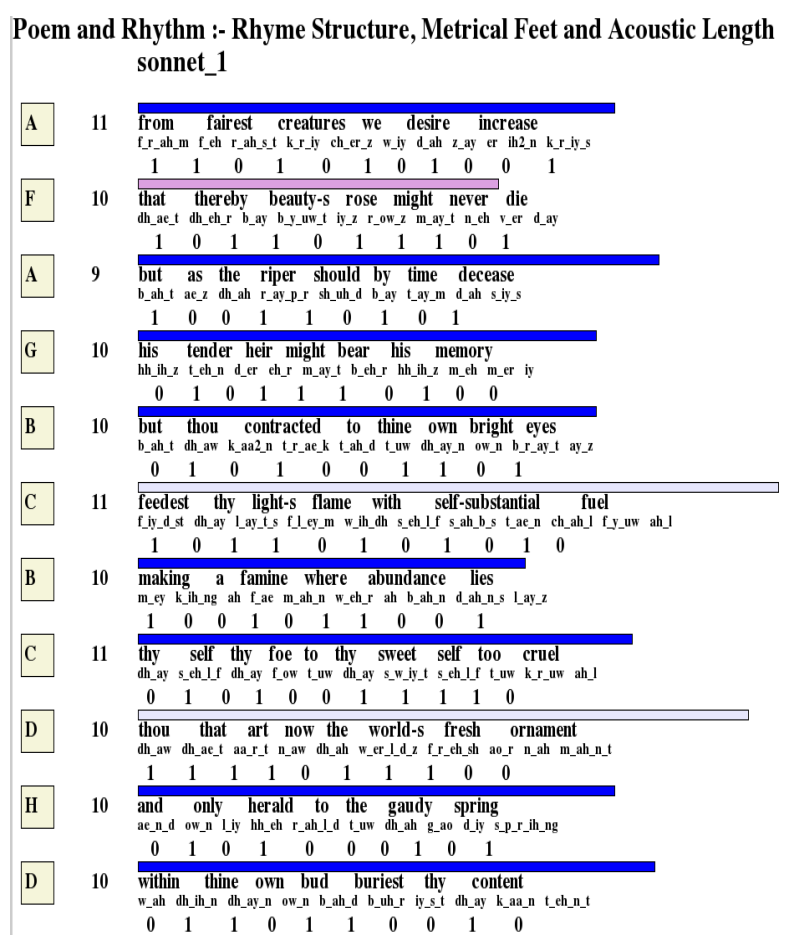

The second set of views is the Poetic Relations View. It is obtained by a single graphical map which however condenses five different levels of

\footnotetext{
${ }^{6}$ Area low: ae aa ah ao aw ay; area high front: iy ih ia y; area high back: uw uh ow ua w; area middle: er ea ax oh eh ey oy. Voiced consonants: $1 \mathrm{~m} \mathrm{n} \mathrm{ng} \mathrm{r} \mathrm{z} \mathrm{zh} \mathrm{dh} \mathrm{d} \mathrm{b} \mathrm{g} \mathrm{v}$ jh; unvoiced consonants: $p \mathrm{~s}$ sh th $\mathrm{h}$ hh $\mathrm{f}$ ch $\mathrm{t}$ k. Obstruents: $\mathrm{t} d \mathrm{~b} \mathrm{p} \mathrm{g} \mathrm{k} \mathrm{jh}$ ch; continuants: $\mathrm{fv}$ z zh s sh th dh h hh; sonorants: $1 \mathrm{~m} \mathrm{n} \mathrm{ng} \mathrm{r.}$
} 
analysis. The Rhyming Structure is obtained by matching line endings in its phonetic form. The result is an capital letter associated with each line on left. This is accompanied by a metrical measure indicating the number of syllables contained in the line. Then the text of the poem and underneath the phonetic translation at syllable level. Finally, another annotation is added mapping syllable type with sequences of $0 / 1$. This should serve a metrical analysis which can be accomplished by the user completed by comments on poetry type which will be presented at the conference. The additional important layer of analysis that this view makes available is an acoustic phonetic image of each line represented by a coloured streak computed on the basis of the average syllable length in msec derived from our database of syllables of British English.

Finally, the third set of views, the Semantic Relational View, produced by the modules of the system derived from VENSES (Delmonte et al., 2005). This view is organized around four separate graphical poetic maps: a map which highlights Event and State words in the poem; a map which highlights Concrete vs Abstract words. Both these two maps address nouns and adjectives. They also indicate Affective and Sentiment analysis (Delmonte \& Pallotta, 2011; Delmonte 2014), an evaluation related to nouns and adjective - which however will be given a separate view when the Appraisal-based dictionary will be completed at the conference. A map which contains main Topics, Anaphoric and Metaphoric relations, and a final map with Predicate-arguments relations.

Poem and Semantics :- PredicateArgument Relations and Event Anaphora sonnet1

SUBJECT-ARG OBJECT-ARG EVENT-ANAPHORA

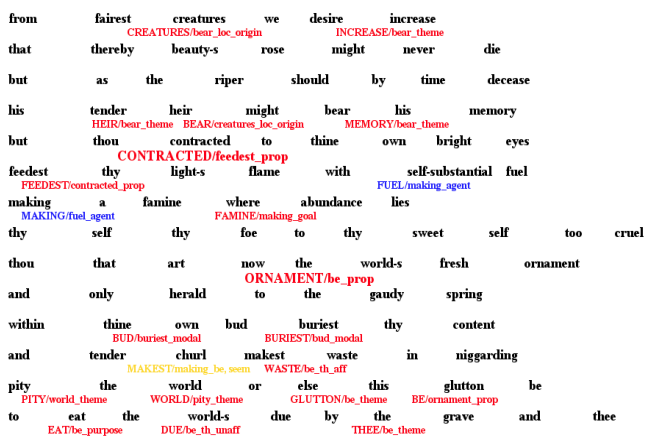

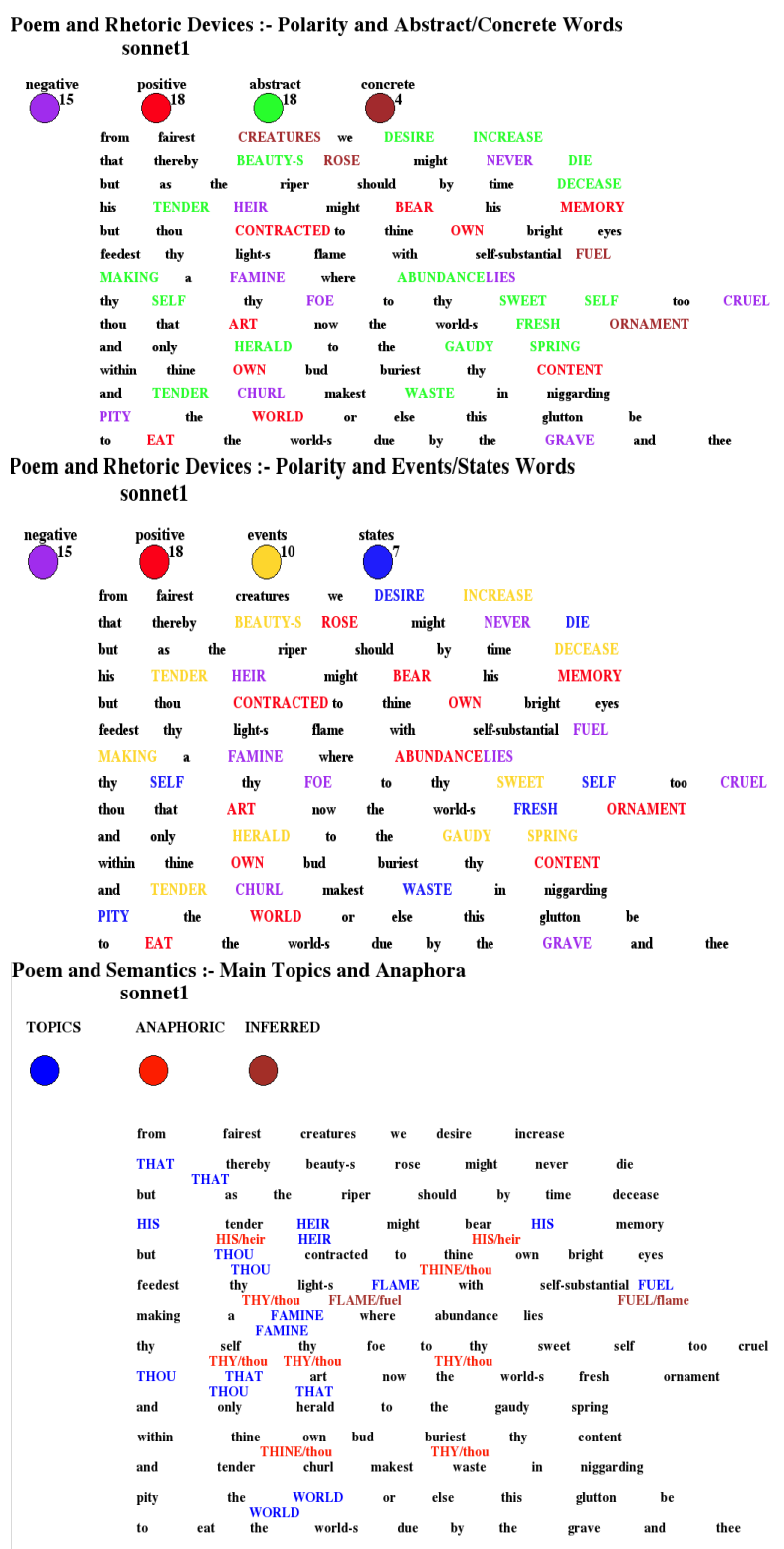

We will now compare a love sonnet like Sonnet 1 to Sonnet 60, which like many other similar sonnets depicts the condition of man condemned to succumb to the scythe of Time - the poet though, will survive through his verse. Here we will restrict ourselves to showing only part of the maps and omit less relevant ones.

In the Phonetic Relations Views the choice of words is strongly related to the main theme and the result is a gloomier, hasher overall sound quality of the poem: number of unvoiced is close to that of voiced consonants; in particular, number of obstruents is higher than the sum of sonorants and continuants. As to Assonances, we see that even 
though A and E sounds - that is open and middle vowels - constitute the majority of sounds, there is a remarkable presence of back and high front vowels. Proportions are very different from what we found in Sonnet 1: $18 / 49$, i.e. dark are one third of light, compared to 21/46, almost half the amount. Also consider number of Obstruents which is higher than number of Sonorants and Continuants together: in Sonnet 1 it was identical to number of Continuants.

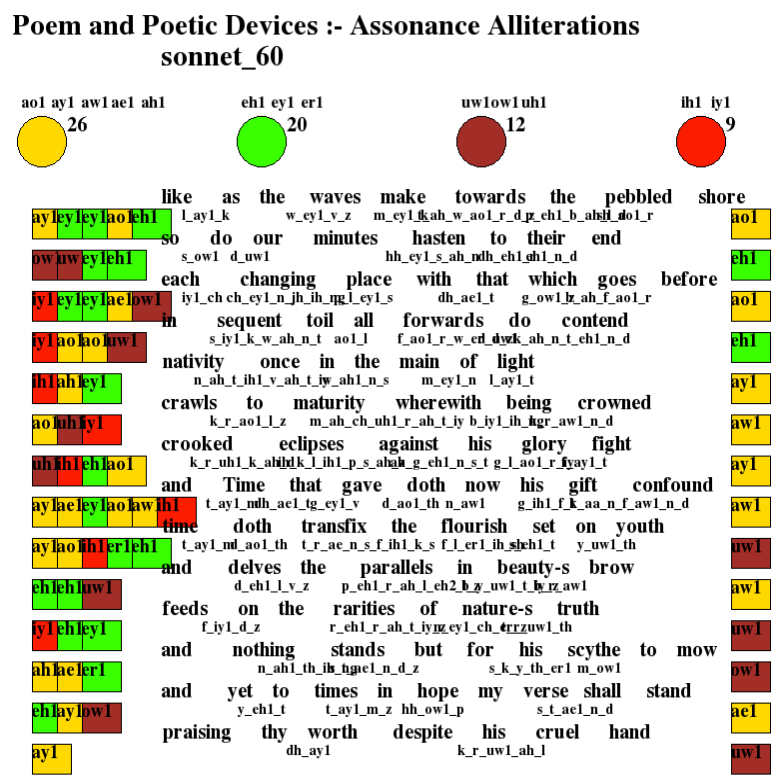

Poem and Poetic Devices :- Phonetic Voiced/Unvoiced Map sonnet_60

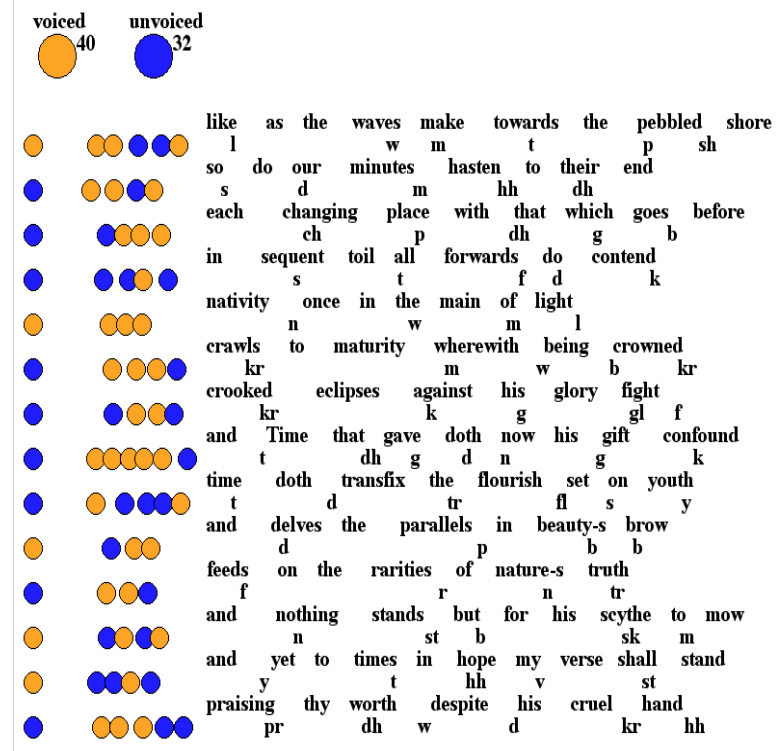

Similar remarks can be made on the map of Unvoiced/Voiced opposition, where we see that we go from 29/43 that is dark sounds are a little bit more than half light ones in Sonnet 1, to $32 / 40$ in Sonnet 60 , i.e. they are almost the same amount.

Eventually, the information coming from affective analysis confirms our previous findings: in Sonnet 1 we see a majority of positive words/propositions, $18 / 15$; the opposite applies to Sonnet 60 , where the ratio is reversed $10 / 21$.
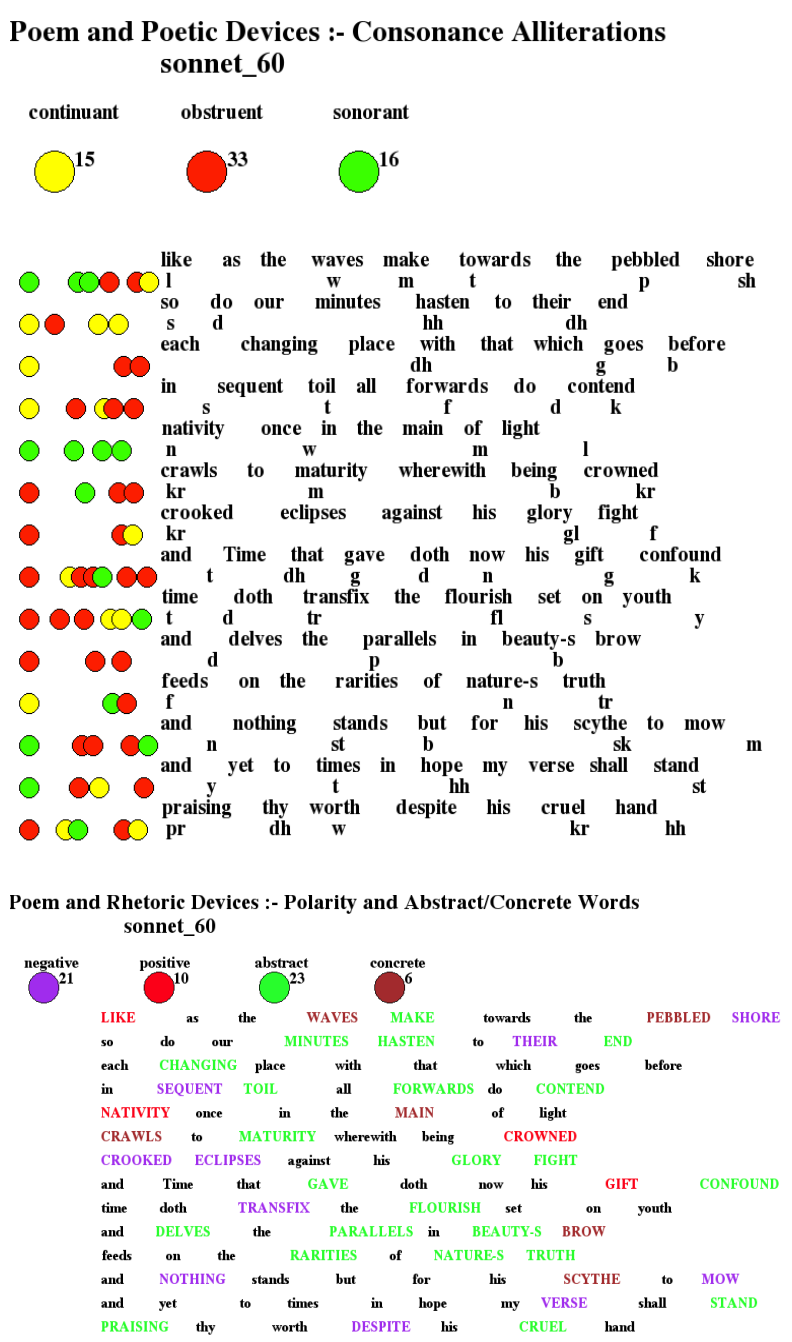

This interpretation of the data is expected also for other poets and is proven by Sylvia Plath's Edge, a poem the author wrote some week before her suicidal death. It's a terrible and beautiful poem at the same time: images of death are evoked and explicitly mentioned in the poem, together with images of resurrection and nativity. The poem starts with an oxymoron: "perfected" is joined with "dead body" and both are predicated of the "woman". We won't be able to show all the maps 
for lack of space, but the overall sound pattern is strongly reminiscent of a death toll. In the Consonances map, there's a clear majority of obstruent sounds and the balance between voiced/unvoiced consonants is in favour of the latter. In the Assonances map we see that dark vowel sounds are almost in same amount of light sounds $28 / 30$.

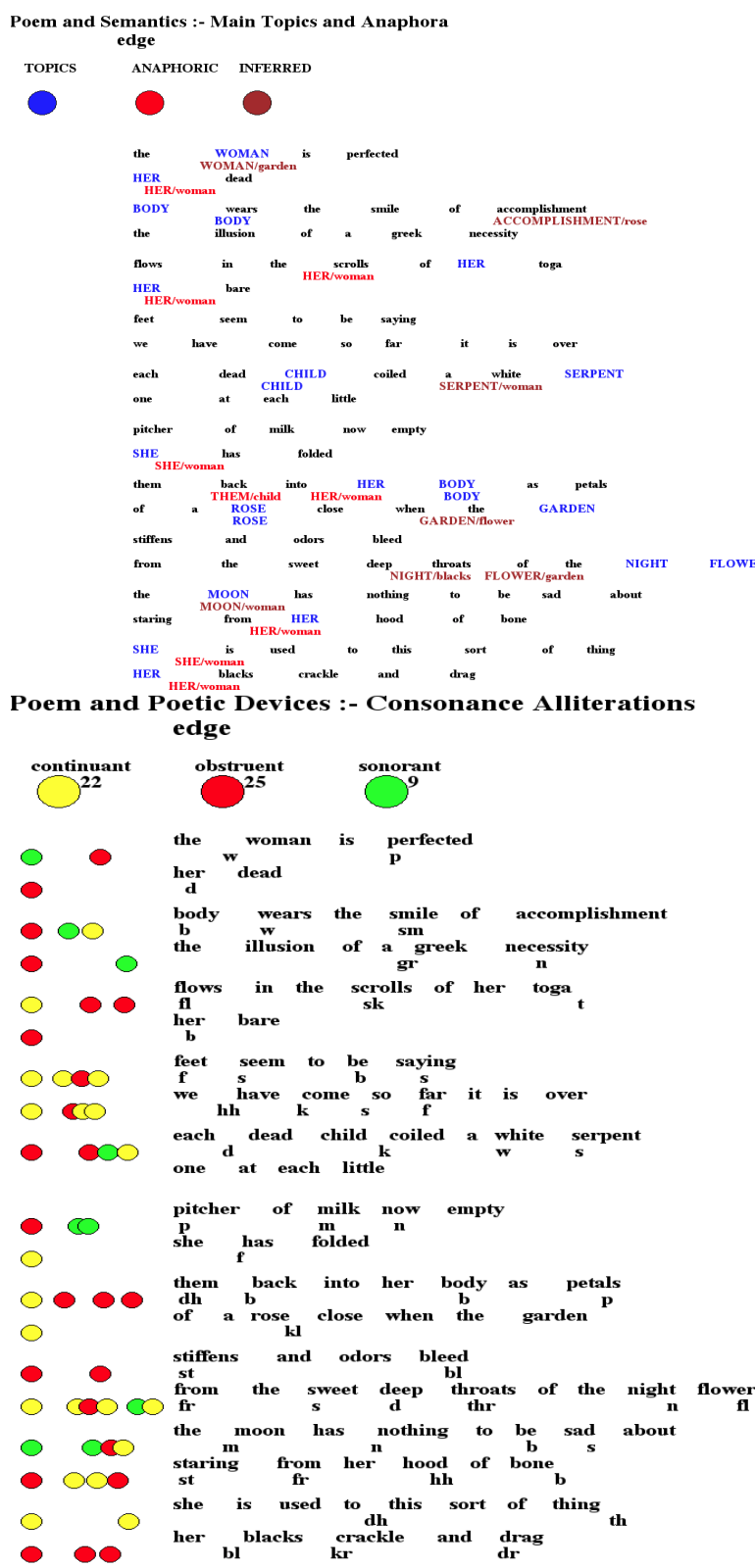

The same applies to the Voiced/Unvoiced distinction which is again in favour of the latter. If we look at the topics and the coherence through anaphora, we find that the main topic is constituted by concepts WOMAN, BODY and CHILD.
There's also a wealth of anaphoric relations expressed by personal and possessive pronouns which depend on WOMAN. In addition, he system has found metaphoric referential links with such images as MOON GARDEN and SERPENT. In particular the Moon is represented as human - "has nothing to be sad about".

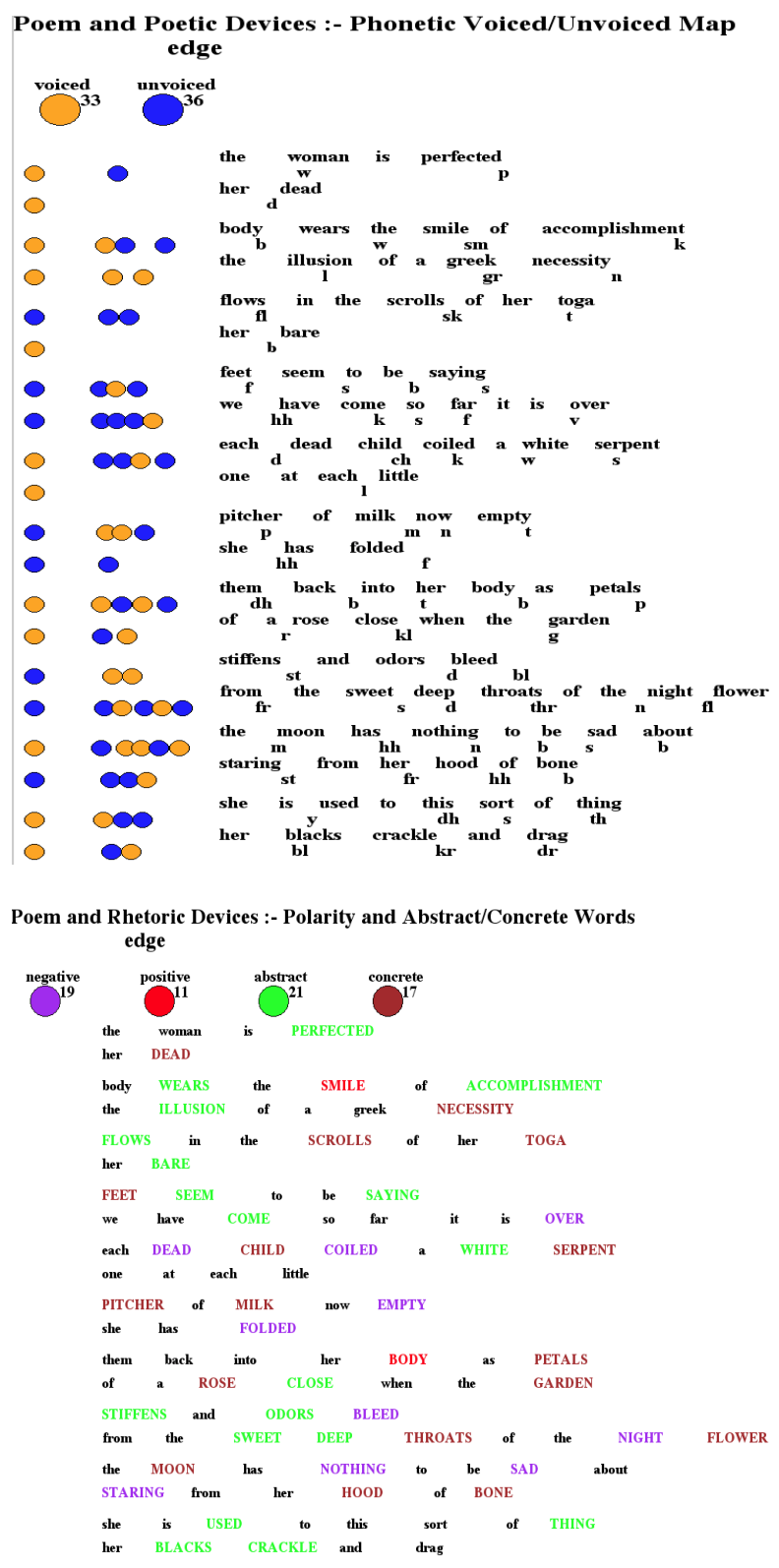

These images are all possible embodiment of the WOMAN, either directly - the Moon is feminine (she) - or indirectly, when the CHILD that the woman FOLDS the children in her BODY, and the children are in turn assimilated to WHITE SERPENTS. 


\section{Computing Mood from the Sonnets}

In this final section we will show data produced by SPARSAR relatively to the relation holding between Mood, Sound and Meaning in half of William Shakespeare's Sonnets. This is done to confirm data presented in the sections above. As will be made clear from Table 1. below, choice of words by Shakespeare has been carefully done in relating the theme and mood of the sonnet to the sound intended to be produced while reading it. Shakespeare's search for the appropriate word is a well-known and established fact and a statistics of his corpus speak of some 29,000 types, a lot more than any English poet whose corpus has been quantitatively analyzed so far (see Delmonte, 2013a).

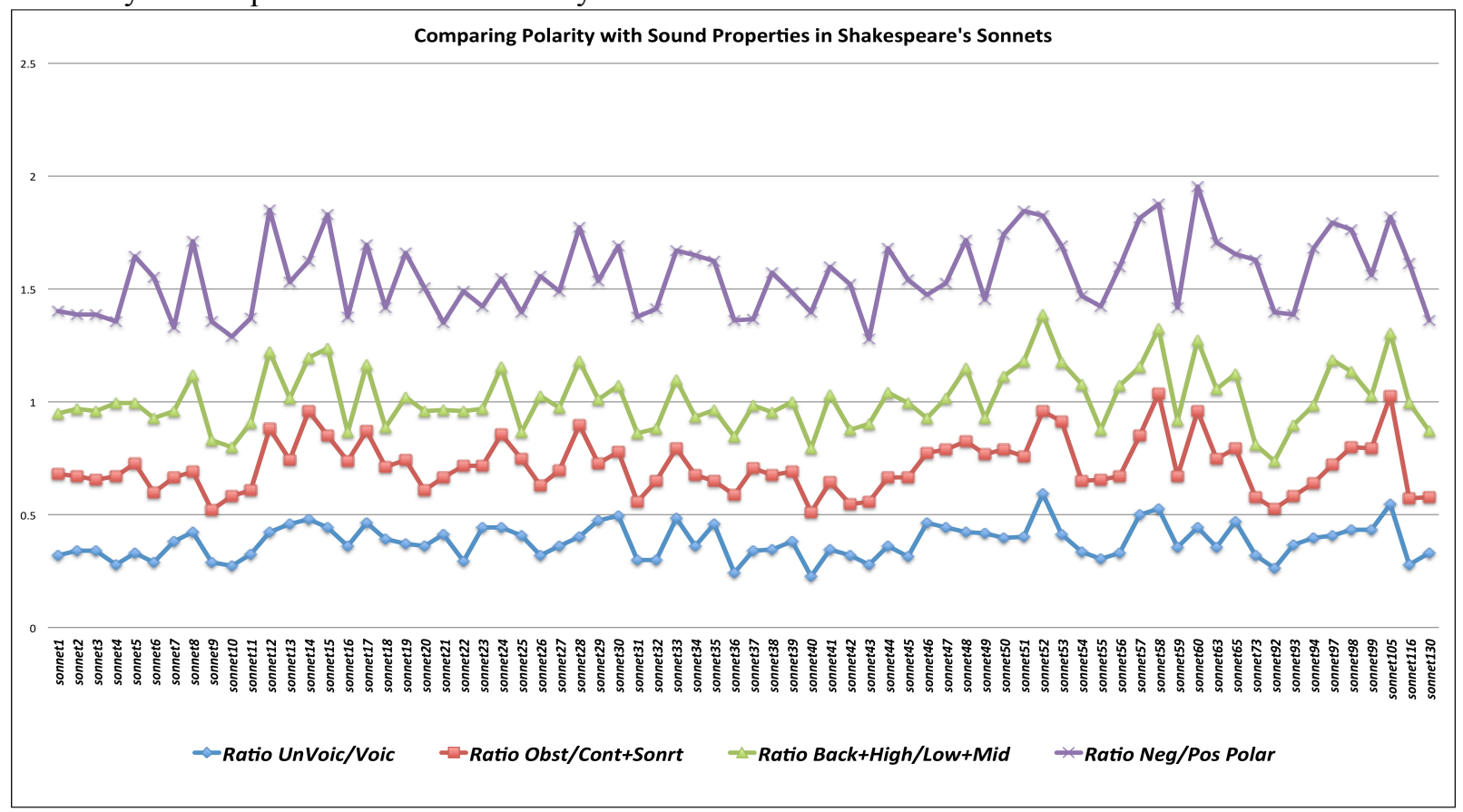

Table 1. Comparing Polarity with Sound Properties of Shakespeare's Sonnets: Blue Line $=$ Ratio of Unvoiced/Voiced Consonants; Red Line $=$ Ratio of Obstruents/Continuants + Sonorants; Green Line $=$ Ratio of Marked Vowels/Unmarked Vowels; Violet Line = Ratio of Negative/Positive Polarity Words/Propositions.

1. sonnets with an overall happy mood; 2 . sonnets about love with a contrasted mood - the lover have betrayed the poet but he still loves him/her, or the poet is doubtful about his friend's love; 3 . sonnets about the ravages of time, the sadness of human condition (but the poet will survive through his verse); 4 sonnets with an overall negative mood. We will look at peaks and dips in the Table 1. and try to connect them to the four possible interpretations of the sonnets.

1. POSITIVE peaks (11): sonnet 6 , sonnet 7 , sonnet 10 , sonnet 16 , sonnet 18 , sonnet 25 , sonnet 26 , sonnet 36 , sonnet 43 , sonnet 116 , sonnet 130

4. NEGATIVE dips (15): sonnet 5, sonnet 8, sonnet 12 , sonnet 14 , sonnet 17 , sonnet 19 , sonnet 28 , sonnet 33 , sonnet 41 , sonnet 48 , sonnet 58 , sonnet 60 , sonnet 63 , sonnet 65 , sonnet 105
2. POSITIVE-CONTRAST (6): sonnet 22, sonnet 24 , sonnet 31 , sonnet 49 , sonnet 55 , sonnet 59

3. NEGATIVE-CONTRAST (1): sonnet 52

Overall, the system has addressed 33 sonnets out of 75 with the appropriate mood selection, $44 \%$. The remaining 42 sonnets have been projected in the intermediate zone from high peaks to low dips.

\section{Conclusion}

We presented a visualization algorithm that works on two XML files, the output of SPARSAR system for poetry analysis. The algorithm decomposes the content of the two XML files into 10 graphical maps whose content can in turn be organized into three macro views that encompass most of a poem's poetic content. In a final section we also verified (successfully) the hypothesis regarding the 
existence of an implicit association between sound and meaning carried by the words making up a poem, by a study of 75 Shakespeare's sonnets. More work needs to be done to improve the Polarity analysis which we intend to project onto the "Appraisal Theory" of meaning. A complete analysis of Shakespeare's sonnets is also under way and will be presented at the conference, together with a comparison with the work of more recent poets.

\section{References}

Agirrezabal Manex, Bertol Arrieta, Aitzol Astigarraga, Mans Hulden, 2013. POS-tag based poetry generation with WordNet, Proceedings of the 14th European Workshop on Natural Language Generation, pages 162-166.

Baayen R. H., R. Piepenbrock, and L. Gulikers. 1995. The CELEX Lexical Database (CD-ROM). Linguistic Data Consortium.

Bacalu C., Delmonte R. 1999. Prosodic Modeling for Syllable Structures from the VESD - Venice English Syllable Database, in Atti $9^{\circ}$ Convegno GFS-AIA, Venezia.

Bacalu C., Delmonte R. 1999. Prosodic Modeling for Speech Recognition, in Atti del Workshop AI*IA "Elaborazione del Linguaggio e Riconoscimento del Parlato", IRST Trento, pp.45-55.

Brysbaert, M., Warriner, A.B., \& Kuperman, V. 2014. Concreteness ratings for 40 thousand generally known English word lemmas. Behavior Research Methods, 46, 904-911.

Byrd Roy J. and M. S. Chodorow. 1985. Using an online dictionary to find rhyming words and pronunciations for unknown words. In Proceedings of the 23rd Annual Meeting of ACL, 277-283.

Delmonte R., 2013a. Transposing Meaning into Immanence: The Poetry of Francis Webb, in Rivista di Studi Italiani, Vol. XXX1, $n^{\circ} 1,835-892$.

Delmonte R., et al. 2005. VENSES - a LinguisticallyBased System for Semantic Evaluation, in $\mathrm{J}$. Quiñonero-Candela et al.(eds.), Machine Learning Challenges. LNCS, Springer, Berlin, 344-371.

Delmonte R. and V. Pallotta, 2011. Opinion Mining and Sentiment Analysis Need Text Understanding, in "Advances in Distributed Agent-based Retrieval Tools", Springer, 81-96.

Delmonte R. \& C. Bacalu. 2013. SPARSAR: a System for Poetry Automatic Rhythm and Style AnalyzeR, SLATE 2013 - Demonstration Track, Grenoble.

Delmonte R. 2013b. Computing Poetry Style, in C. Battaglino, C. Bosco, E. Cambria, R. Damiano, V. Patti, P. Rosso (eds.), Proceeding ESSEM - Emotion and Sentiment in Social and Expressive Media: approaches and perspectives from AI (ESSEM 2013), CEUR Workshop Proceedings, Torino, 148155, http://ceur-ws.org/Vol-1096/.

Delmonte R. \& A.M. Prati. 2014. SPARSAR: An Expressive Poetry Reader, Proceedings of the Demonstrations at the 14th Conference of the EACL, Gotheborg, 73-76.

Delmonte R. 2014. A Computational Approach to Poetic Structure, Rhythm and Rhyme, in R. Basili, A. Lenci, B. Magnini (eds), Proceedings of CLiC-it The First Italian Conference on Computational Linguistics, Pisa University Press, Vol.1, 144-150.

Delmonte R. 2014. ITGETARUNS A Linguistic RuleBased System for Pragmatic Text Processing, in C. Bosco, P. Cosi, F. Dell'Orletta, M. Falcone, S. Montemagni, Maria Simi (eds.), Proceedings of Fourth International Workshop EVALITA, Pisa University Press, Vol. 2, 64-69.

Delmonte R., 2015. SPARSAR - Expressivity in TTS and its Relations to Semantics, Invited Talk at AISV 2015, Bologna.

Genzel Dmitriy, J. Uszkoreit, and F. Och. 2010. "Poetic" statistical machine translation: Rhyme and meter. In Proceedings of EMNLP.

Fónagy, Iván (1971) "The Functions of Vocal Style", in Seymour Chatman (ed.), Literary Style: A Symposium. London: Oxford UP, 159-174.

Gérvas, P. (2001). An expert system for the composition of formal Spanish poetry. Knowledge-Based Systems, 14(3):181-188.

Gérvas, P. (2010). Engineering linguistic creativity: Bird flight and jet planes. In Proceedings of the NAACL HLT 2010 Second Workshop on Computational Approaches to Linguistic Creativity, pages $23-30$.

Greene E., T. Bodrumlu, K. Knight. 2010. Automatic Analysis of Rhythmic Poetry with Applications to Generation and Translation, in Proceedings of the 2010 Conference on EMNLP, 524-533.

Jakobson, R. 1978. Six lectures on sound and meaning (Trans.: J. Mepham). Cambridge: MIT Press (Original work published in 1976).

Jakobson, R., \& Waugh, L. 1978. The sound shape of language. Bloomington: Indiana University Press.

Kao Justine and Dan Jurafsky. 2012. "A Computational Analysis of Style, Affect, and Imagery in Contemporary Poetry". in Proc. NAACL Workshop on Computational Linguistics for Literature.

Keppel-Jones David. 2001. The Strict Metrical Tradition: Variations in the Literary Iambic Pentameter from Sidney and Spenser to Matthew Arnold, Mcgill Queens Univ. Pr., 280.

Manurung Hisar Maruli, G. Ritchie, and H. Thompson. 2000. Towards a computational model of poetry generation. In Proceedings of AISB Symposium on 
Creative and Cultural Aspects and Applications of AI and Cognitive Science, 17-20.

Manurung M.H., G. Ritchie, H. Thompson. 2000. A Flexible Integrated Architecture For Generating Poetic Texts. in Proceedings of the Fourth Symposium on Natural Language Processing (SNLP 2000), Chiang Mai, Thailand, 7-22.

Macdermott M.M. 1940. Vowel Sounds in Poetry: Their Music and Tone Colour, Psyche Monographs, No.13, London: Kegan Paul, 148 pp.

Mazzeo, M. 2004. Les voyelles colorées: Saussure et la synesthésie. Cahiers Ferdinand de Saussure, 57,129143.

Mohammad Saif, Colourful Language: Measuring Word-Colour Associations, 2011a. In Proceedings of the ACL 2011 Workshop on Cognitive Modeling and Computational Linguistics (CMCL), June 2011, Portland, OR.

Mohammad Saif, Even the Abstract have Colour: Consensus in Word Colour Associations, 2011b. In Proceedings of the 49th Annual Meeting of the Association for Computational Linguistics: Human Language Technologies, June 2011, Portland, OR.

Sonderegger Morgan. 2011. Applications of graph theory to an English rhyming corpus. Computer Speech and Language, 25:655-678.

Sravana Reddy \& Kevin Knight. 2011. Unsupervised Discovery of Rhyme Schemes, in Proceedings of the 49th Annual Meeting of ACL: shortpapers, 77-82.

Toivanen Jukka, Hannu Toivonen, Alessandro Valitutti, \& Oskar Gross, 2012. Corpus-based generation of content and form in poetry. In Proceedings of the Third International Conference on Computational Creativity.

Tsur, Reuven. 1992. What Makes Sound Patterns Expressive: The Poetic Mode of Speech-Perception. Durham N. C.: Duke UP.

Tsur Reuven. 1997a. "Poetic Rhythm: Performance Patterns and their Acoustic Correlates". Versification: An Electronic Journal Devoted to Literary Prosody. (http://sizcoll.u-shizuokaken.ac.jp/versif/Versification.html)

Tsur Reuven. 2012. Poetic Rhythm: Structure and Performance: An Empirical Study in Cognitive Poetics, Sussex Academic Press, 472. 